\title{
Pancreatic cancer therapy with a novel pump for controlled drug release
}

\author{
MARI HARAMOTO $^{1}$, MASAYUKI KOHNO $^{1}$, OUMI NAKAJIMA ${ }^{1}$, TOMOHISA HORIBE ${ }^{1}$, \\ MASANOBU KIYOHARA ${ }^{2}$, HIROHIDE FUKAZAWA ${ }^{2}$, TSUYOSHI TOGAWA ${ }^{2}$ and KOJI KAWAKAMI ${ }^{1}$ \\ ${ }^{1}$ Department of Pharmacoepidemiology, Graduate School of Medicine and Public Health, Kyoto University, \\ Yoshidakonoecho, Sakyoku, Kyoto 606-8501; ${ }^{2}$ Olympus, Co., Shinjuku Monolith, \\ 3-1 Nishishinjuku 2-chome, Shinjuku-ku, Tokyo 163-0914, Japan
}

Received July 13, 2009; Accepted September 30, 2009

DOI: $10.3892 /$ or_00000644

\begin{abstract}
Enhancing antitumor activity and minimizing treatment side effects are important issues in cancer therapy. One method to deal with these issues is the utilization of a drug delivery system (DDS). In this study, we developed a novel drug administration pump, a mechanically controlled DDS (M-DDS). The antitumor activity of 5-fluorouracil (5-FU) (15 or $30 \mathrm{mg} / \mathrm{kg} /$ day) was evaluated in comparison with systemic intraperitoneal (i.p.) administration for 7 days in a rat model of human pancreatic cancer. The M-DDS was superior to i.p. administration in enhancing antitumor activity and also prolonging median survival from 69 to 85 days at the lower drug dose $(15 \mathrm{mg} / \mathrm{kg} /$ day $)$. In addition, toxicities in liver, kidney and spleen were found in animals receiving i.p. administration, whereas rats receiving M-DDS treatment did not show these toxicities. The concentration of 5-FU in tumors 1 day after the completion of treatment was considerably higher in rats receiving M-DDS treatment. These results suggest that this novel M-DDS may be a powerful tool for the treatment of pancreatic cancer in combination with conventional chemotherapeutic drugs, offering strong antitumor activity with fewer toxicities. This novel M-DDS, consisting of a control circuit and drug reservoir/pump unit, may be a useful tool for the treatment not only of pancreatic cancer but also of various other accessible cancers for which there is no effective treatment, such as bile-duct and brain tumors.
\end{abstract}

\section{Introduction}

The number of patients with pancreatic cancer continues to increase annually, with $\sim 37,170$ new cases reported in the USA

Correspondence to: Dr Koji Kawakami, Department of Pharmacoepidemiology, Graduate School of Medicine and Public Health, Kyoto University, Yoshidakonoecho, Sakyoku, Kyoto 606-8501, Japan

E-mail: kawakami-k@umin.ac.jp

Key words: medical device, pancreatic cancer, mechanical drug delivery system, 5-fluorouracil, side effect in 2007 (1). In the USA, pancreatic cancer has become one of the leading causes of death from malignant neoplasm (ranking fifth in 2005) due to the following reasons. First, early detection of pancreatic cancer is difficult and it is generally discovered as a progressive tumor which is not excisable. Second, no aggressive therapies, including antitumor drugs, are available for the treatment of advanced disease. Although gemcitabine has been used for stage II, III and IV advanced pancreatic cancer, the 12-month survival rate for this disease is still $<18 \%$ (2). Thus, pancreatic cancer is one of the most difficult diseases to cure. These facts led us to develop a new tool for the treatment of this disease.

An effective drug delivery system (DDS) is one of the keys to the success of desirable antitumor therapy. To avoid the unwanted effects caused by antitumor drugs, molecularly targeted drugs including monoclonal antibodies (3) targeted small molecules (4), cancer vaccines (5) and immunotoxins $(6,7)$ have been developed. In addition, an effective DDS is necessary for delivering conventional antitumor drugs to enhance their antitumor activity and to decrease unwanted side effects. Several groups have reported a variety of DDSs, using such methods as liposomes $(8)$, mechanical devices $(9,10)$ and atelocollagen (11), for use in not only cancer therapy but also several other diseases. Previously, we demonstrated that an osmotic infusion pump inserted into small animals allowed continuous infusion of drug at a constant rate (12). However, the infusion rate could not be controlled during treatment because the pump was not powered by a control circuit and a superior device was necessary for more effective drug administration (12).

In this study, we developed a novel, local drug infusion device for the treatment of progressive pancreatic cancer. This device is a mechanically controlled DDS (M-DDS) with the ability for long-term drug release by use of a powered pump. The effect of the widely used conventional anticancer drug 5-fluorouracil (5-FU) was evaluated in combination with this device, compared with systemic intraperitoneal (i.p.) administration of 5-FU. Our results suggest that the new M-DDS is superior in the delivery of an antitumor effect in pancreatic cancer. In addition, the safety profile of organs and blood laboratory test results from experimental animals demonstrated the excellence of the M-DDS in comparison with systemic i.p. delivery. 


\section{Materials and methods}

Reagents and cell line. 5-FU was purchased from Sigma (St. Louis, MO, USA). Human pancreatic tumor cell line BxPC-3 was cultured in RPMI-1640 supplemented with $10 \%$ fetal bovine serum, $100 \mu \mathrm{g} / \mathrm{ml}$ penicillin and $100 \mu \mathrm{g} / \mathrm{ml}$ streptomycin.

Experimental animals. Male athymic nude rats (weight 200-250 g) purchased from CLEA Japan (Tokyo, Japan) were used in this study. Animals were housed under constant temperature $\left(24 \pm 2^{\circ} \mathrm{C}\right)$ and humidity $(50 \pm 10 \%)$ with a controlled $12 \mathrm{~h}$ light/12 h dark cycle. All experiments were carried out in accordance with the guidelines of the Kyoto University School of Medicine.

Orthotopic model of human pancreatic cancer. A rat model of orthotopically xenografted human pancreatic tumor was developed as described previously (6). Athymic nude rats were anesthetized and placed in the supine position. The pancreas was exteriorized from a left lateral abdominal incision. The tumor pieces $(5 \times 5 \times 5 \mathrm{~mm})$ obtained from subcutaneously growing tumors derived from BxPC-3 cells were transplanted to the body of the pancreas, ligating with absorbable surgical sutures.

$M-D D S$. A photograph of the prototype M-DDS is shown in Fig. 1A. The device consists of a control circuit, drug reservoir, and pump unit. Drug release by the pump is modulated by the control circuit and drug in the reservoir is designed to infuse continuously into the tumor. When a tumor piece was transplanted into the pancreas of a rat, a catheter was also inserted into the tumor to administer the drug (Fig. 1B). The M-DDS device was then immobilized on the back of the rat and the catheter connected to the drug reservoir (Fig. 1C).

Animal studies. Athymic nude rats were transplanted orthotopically with pancreatic tumor on day 0 . In the systemic treatment group nude rats were treated with 15 or $30 \mathrm{mg} / \mathrm{kg} / \mathrm{day}$ 5-FU by i.p. administration for 7 days (one injection per day, from day 0 to day 6 ) and in the M-DDS treatment group rats received $5-\mathrm{FU}$ (15 or $30 \mathrm{mg} / \mathrm{kg}$ total infusion per day, from day 0 to day 7) by continuous infusion with M-DDS. 5-FU doses employed were selected according to the previous study optimized for 5-FU treatment in animal model of human pancreatic cancer xenograft (13). A control group received i.p. Tris/ $\mathrm{HCl}(\mathrm{pH} .8 .5)$ alone, because this reagent is used as a solvent for 5-FU. Tumor size was measured by ultrasonography (EU-M30S; Olympus Co., Tokyo, Japan). Tumor volume was calculated using the following formula:

Volume $\left(\mathrm{mm}^{3}\right)=\left([\text { short diameter }(\mathrm{mm})]^{2} \times\right.$ [long diameter $(\mathrm{mm})]) / 2$

In addition, tumor, liver, pancreas, spleen, kidney and blood were collected from rats during and after 5-FU treatment for determination of 5-FU, or one day after the treatment period (day 7 or 8) for histological analysis.

Histological analysis. Organs were fixed in $4 \%$ buffered formaldehyde and paraffin-embedded $5-\mu \mathrm{m}$ sections were stained with hematoxylin and eosin. The images were captured by BX-51 microscope equipped with a DP-25 CCD camera (Olympus Co.).

5-FU detection by HPLC. The amount of 5-FU in blood or each organ homogenate was determined by modified version of a method described previously (14). Organ was homogenized with $0.9 \% \mathrm{NaCl}$ and the homogenates or serum was added to $1 \mathrm{M}$ sodium acetate buffer ( $\mathrm{pH} 4.8$ ) and $20 \%$ anhydrous sodium sulfate. The mixture was shaken in ethyl acetate and centrifuged and the organic layer was collected and dried. The resultant samples $(10 \mu \mathrm{l})$ were injected onto the column of a high performance liquid chromatography (HPLC) system (GL-7410, GL Sciences, Tokyo, Japan) with a variable wavelength UV detector (GL-7451, GL Sciences) to detect the 5-FU level.

Statistical analysis. All values are expressed as the mean \pm SD and statistical analysis was calculated by a one-way ANOVA with Dunnett test. Differences were considered to be significant at $\mathrm{P}<0.01$.

\section{Results}

Antitumor activity of 5-FU against orthotopic pancreatic tumor. First we compared the antitumor activity of 5-FU given by systemic and M-DDS administration. Ultrasonic echo was utilized to measure tumor size (Fig. 2A). As shown in Fig. 2B, pancreatic tumors without treatment (control group) grew the most and mean tumor size reached $4678 \pm 637 \mathrm{~mm}^{3}$ by day 30. On the other hand, tumor size in rat receiving M-DDS infusion was significantly smaller compared to the controls: $1954 \pm 358 \mathrm{~mm}^{3}$ in the $15 \mathrm{mg} / \mathrm{kg} \mathrm{M}-D D S$ group and $1479 \pm 384 \mathrm{~mm}^{3}$ in the $30 \mathrm{mg} / \mathrm{kg}$ M-DDS group. In contrast, with systemic administration of 5-FU, although tumor size in rats receiving the higher dose of treatment $(30 \mathrm{mg} / \mathrm{kg}$ ) demonstrated significant tumor suppression by day 30 $\left(2269 \pm 536 \mathrm{~mm}^{3}\right)$, the lower dose of $5-\mathrm{FU}(15 \mathrm{mg} / \mathrm{kg})$ failed to demonstrate antitumor activity to $\mathrm{BxPC}-3$ tumors $\left(4384 \pm 415 \mathrm{~mm}^{3}\right)$. These results suggest that treatment via M-DDS is superior to conventional systemic administration of anticancer drug and provides optimal antitumor activity against pancreatic tumors.

Treatment via M-DDS prolongs survival of animals with pancreatic tumor. Next we assessed survival of animals harboring BxPC-3 pancreatic tumor treated with 5-FU by i.p. or M-DDS infusion for 7 days $(15 \mathrm{mg} / \mathrm{kg})$. As shown in Fig. 3, control animals started dying from day 40, and all the animals died by day 89 . Median survival time for the control group was 66 days (range, 40-89 days). Interestingly, systemic i.p. treatment of animals demonstrated shorter median survival time (59 days; range 50-87 days), whereas the median survival time of animals receiving treatment via M-DDS was considerably greater (85 day; range, 54-90 days) and four animals remained alive for $>100$ days. These results suggest that M-DDS infusion of 5-FU prolongs survival of animals bearing pancreatic tumors.

Drug localization in organs. Because M-DDS infusion of drug was considered to be superior to systemic i.p. administration 

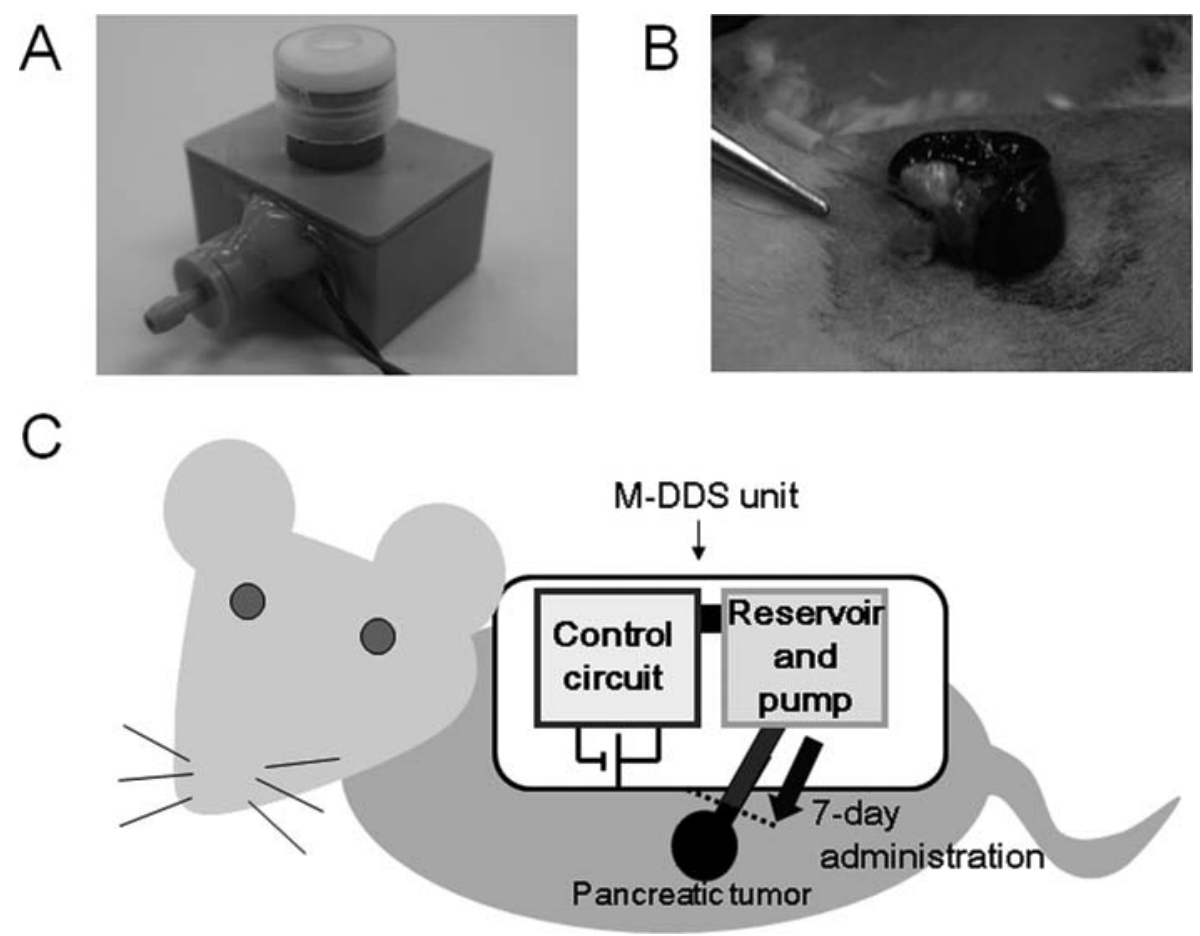

Figure 1. The M-DDS and implantation into the experimental animals. (A) Drug reservoir/pump unit and control circuit of the M-DDS device. (B) Implanting of a catheter for drug infusion into a pancreatic tumor. (C) Schematic diagram to show the M-DDS immobilized on the back of a rat orthotopically transplanted with human tumor.
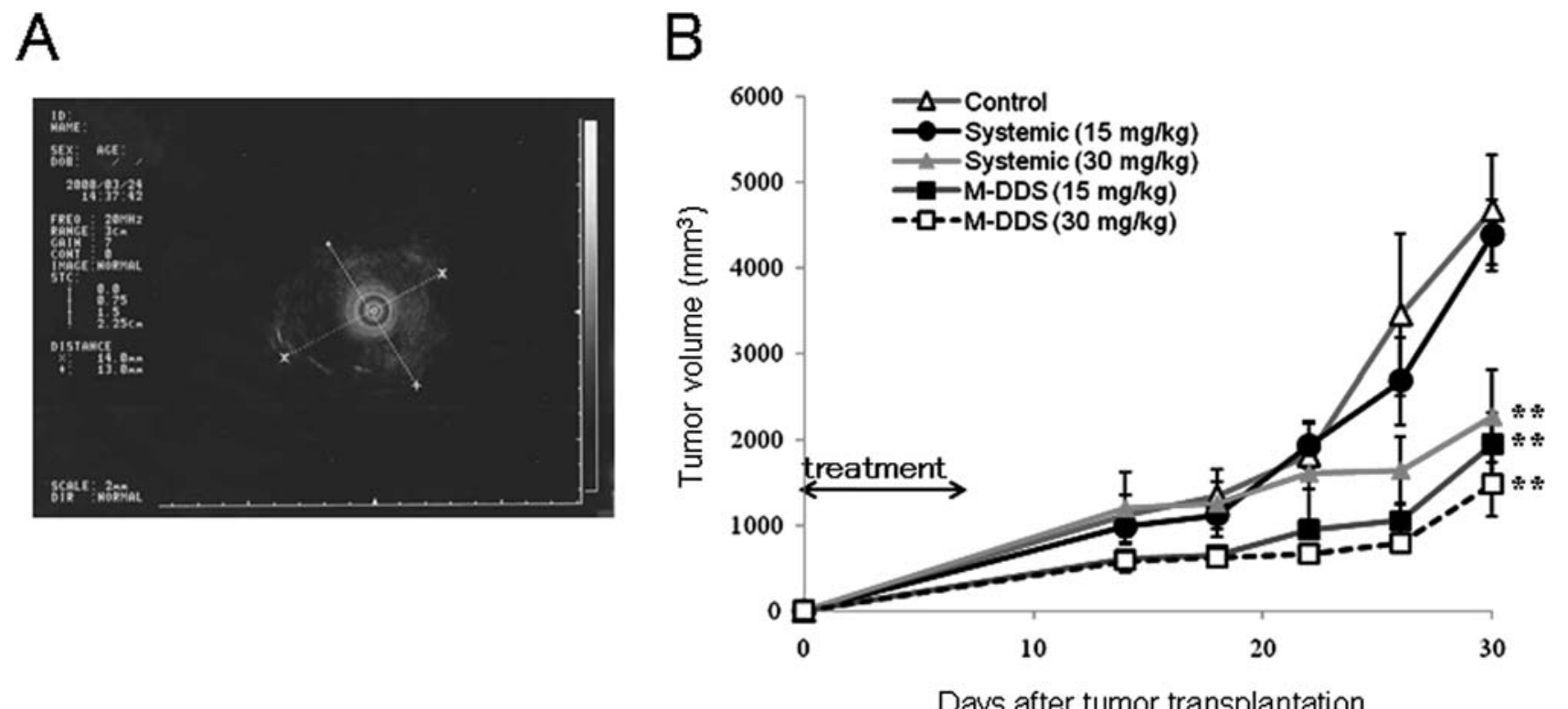

Figure 2. Drug administration by M-DDS induces effective antitumor activity in pancreatic tumor. (A) Tumor diameters were determined using ultrasonography. (B) Rats orthotopically transplanted with BxPC-3 pancreatic tumor were treated with 15 or $30 \mathrm{mg} / \mathrm{kg} / \mathrm{day} 5$-FU for 7 days by either i.p. or M-DDS infusion. Each group consisted of nine animals and experiments were repeated three times; ${ }^{* *} \mathrm{P}<0.01$ vs. control.

in terms of both antitumor activity and survival, we then assessed the 5-FU content of tumor and liver, pancreas, spleen, kidney and blood. HPLC analysis was used to determine drug localization. Tumor and organs of animals receiving 5-FU (30 mg/kg) for 7 days by i.p. or M-DDS infusion were collected on days 1, 3, 7 and 8. As shown in Fig. 4A, 5-FU level in tumor of animals receiving M-DDS infusion was considerably higher than in animals treated by i.p. infusion. 5-FU was highest on day 7 and decreased by day 8 .

In all the organs assessed, 5-FU level was markedly lower in animals receiving M-DDS infusion when compared with i.p. systemic treatment (Fig. 4B-F). In animals receiving i.p. 5-FU treatment, detectable levels of 5-FU were found in liver (Fig. 4B), normal pancreas (Fig. 4C), spleen (Fig. 4D), 


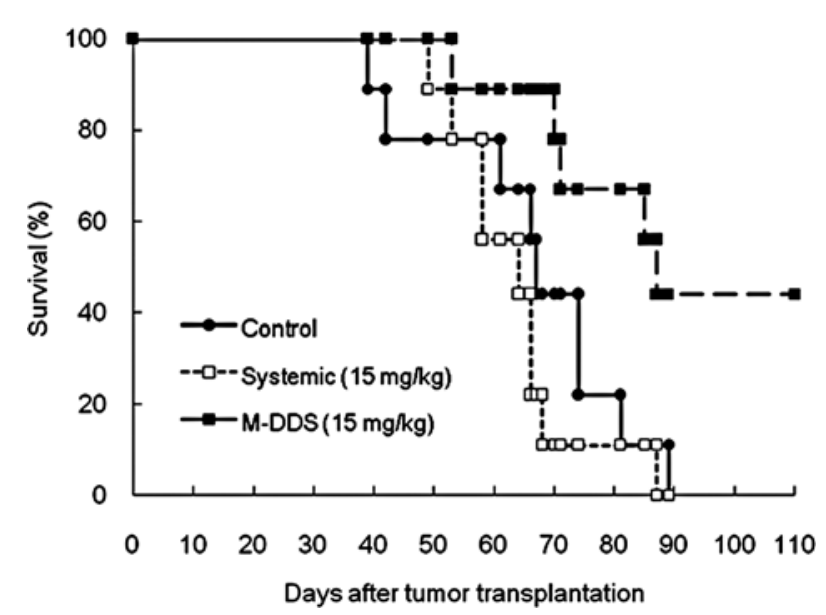

Figure 3. Survival of rats bearing orthotopic pancreatic tumor. BxPC-3 tumor was transplanted into the body of the pancreas in nude rats on day 0 and animals received 5-FU by either i.p. route or M-DDS (15 mg/kg/day) for 7 days. Survival rates are expressed as a percentage of surviving rats compared with day 0 for each group. Each group consisted of 9 animals and experiments were repeated three times.

and kidney (Fig. 4E) during the treatment period (until day 7). These results suggest that M-DDS infusion effectively limits drug delivery to the target tumor without delivery to normal organs.

Limited organ toxicities induced by M-DDS treatment. To assess organ toxicities in animals treated with 5-FU by either i.p. or M-DDS infusion, histological analysis of vital organs was performed. Samples from liver (Fig. 5A), kidney (Fig. 5B) and spleen (Fig. 5C) were harvested one day after the end of the treatment period.

In animals treated with i.p. 5-FU, toxic changes were observed in liver, kidney and spleen. Livers from animals treated with high dose i.p. 5-FU (30 mg/kg) exhibited a dilatation of vascular congestion in the sinusoids (arrow heads in Fig. 5A) and vena centralis (data not shown). In kidney, dilation of Bowman's space (arrows in Fig. 5B) and degeneration of the renal tubule (arrowheads in Fig. 5B) were found on treatment with $15 \mathrm{mg} / \mathrm{kg}$ i.p. 5-FU and degeneration in splenic white pulp (arrows in Fig. 5C) was found in spleen receiving systemic $30 \mathrm{mg} / \mathrm{kg}$ i.p. 5-FU. In contrast, we did not find any signs of toxicity in the organs (Fig. 5A-C) or in the results from blood serum chemistry tests (data not shown) of animals receiving 5-FU via M-DDS (30 mg/kg dose). These results suggest that possible organ toxicities caused by systemic administration of 5-FU can be avoided by M-DDS infusion of drug for the treatment of pancreatic tumor.

\section{Discussion}

In this study we demonstrated that local continuous administration by M-DDS enhanced the antitumor effect, prolonged survival and decreased organ toxicity by 5-FU. We showed here that a half dose of 5-FU, when given by M-DDS, accomplished the same tumor suppression as a single dose given i.p. daily. Furthermore, it was found that M-DDS infusion of 5-FU prolonged survival of animals bearing pancreatic tumors compared with i.p. systemic treatment. Finally, it was clear that the novel M-DDS delivered 5-FU to the tumor specifically without detectable levels of the drug in

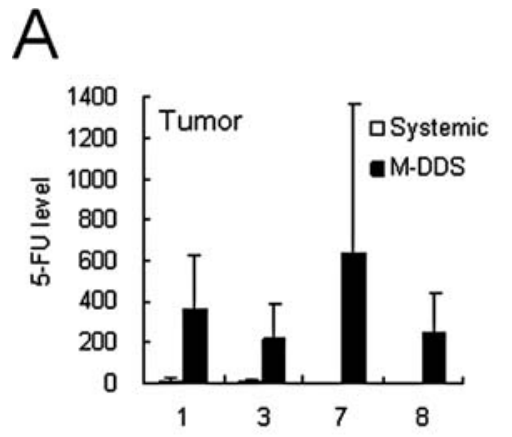

Days after transplantation of tumor

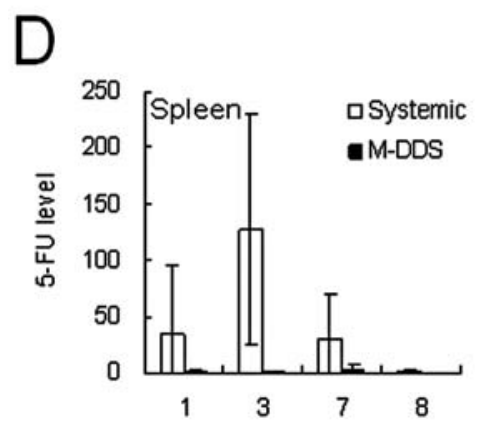

Days after transplantation of turnor

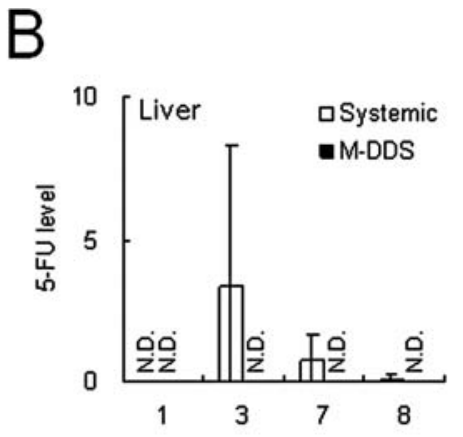

Days after transplantation of tumor

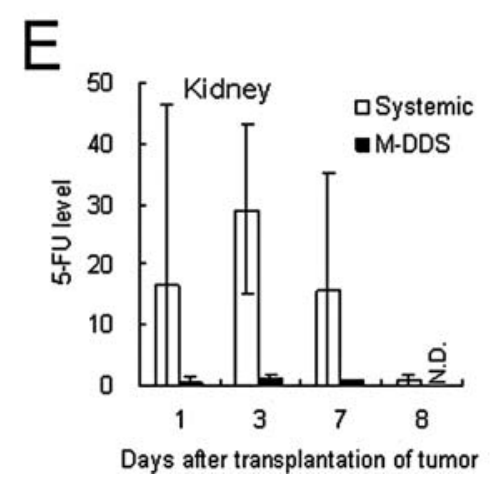

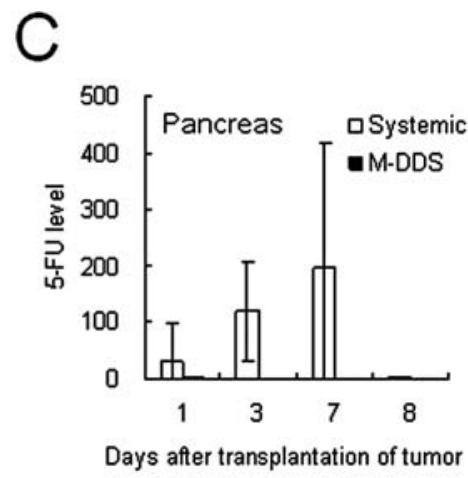

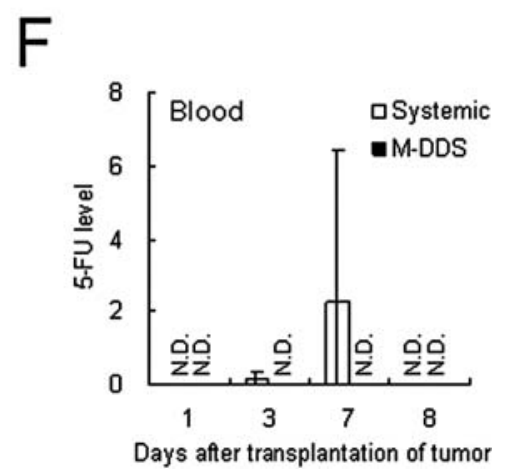

Figure 4. 5-FU levels in tumor and organs. (A) Tumor, (B) liver, (C) pancreas, (D) spleen, (E) kidney and (F) blood were taken from animals bearing orthotopic BxPC-3 pancreatic tumors receiving 5-FU $(30 \mathrm{mg} / \mathrm{kg}$ ) by systemic i.p. or M-DDS infusion for 7 days ( $\mathrm{n}=4)$. Tumor, organ and blood samples were homogenized and 5-FU levels were analyzed using HPLC. Data represent 5-FU level ( $\mu \mathrm{g})$ per gram of tissue. N.D., not detected. 

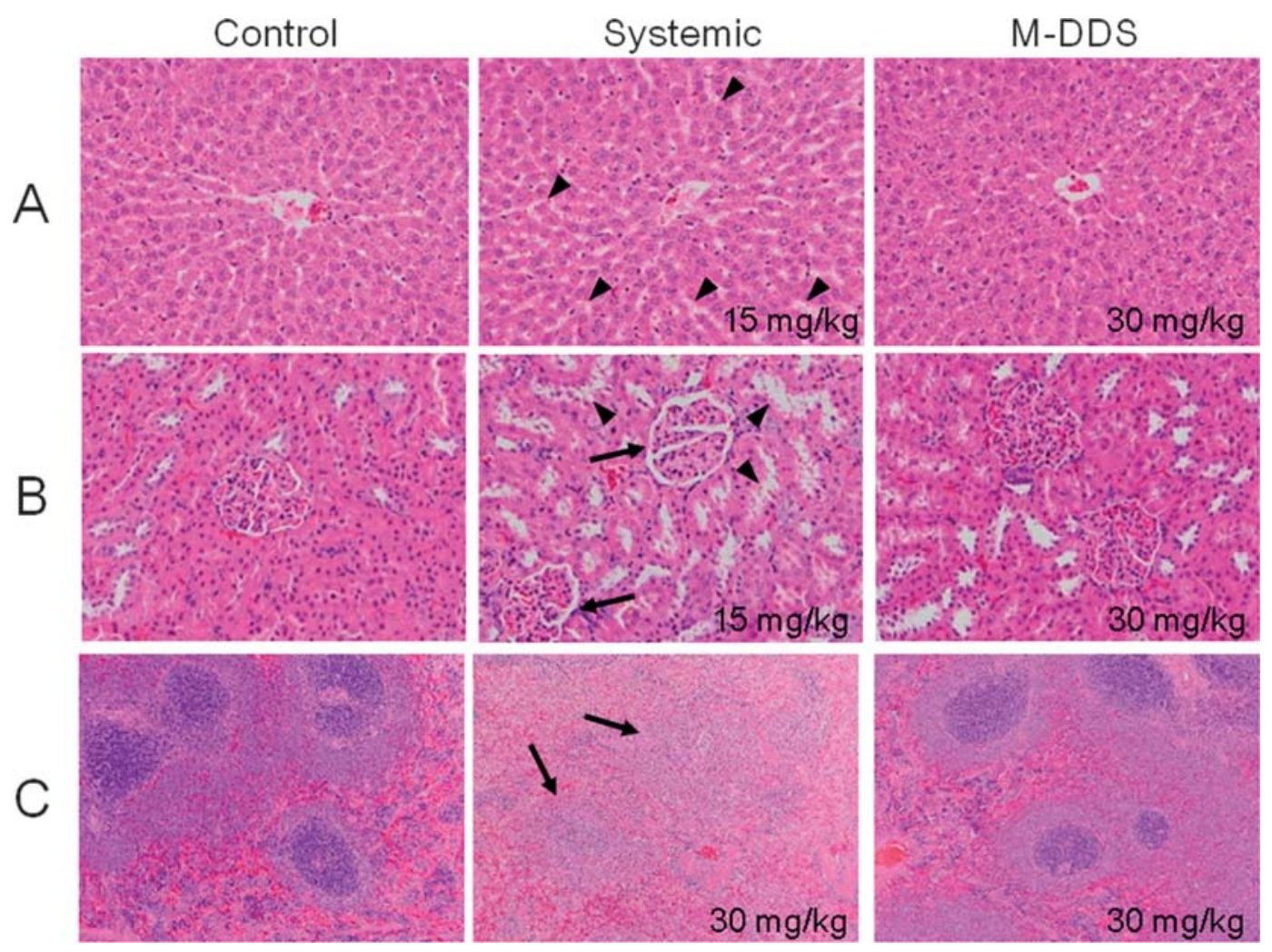

Figure 5. Organ toxicities in animals receiving 5-FU. Rats orthotopically transplanted with BxPC-3 tumor were treated with 5-FU by i.p. or M-DDS infusion (15 or $30 \mathrm{mg} / \mathrm{kg} /$ day) for 7 days. (A) Liver, (B) kidney and (C) spleen were harvested for histological examination. Original magnifications: (A) x200; (B and C) $\mathrm{x} 400$.

other organs and that organ toxicity was attenuated compared with systemic treatment.

5-FU is a typical antimetabolite with a strongly timedependent effect and continuous intravenous infusion is considered to be the optimal treatment regimen (15-17). Administration of 5-FU as a continuous infusion for protracted periods clearly improves the therapeutic index for this agent in patients with advanced colon cancer with respect to the response rate and reduction of toxicity (18). An antimetabolite of 5-FU generates F-deoxy-UMP, which shows antitumor activity, gastrointestinal toxicity and myelotoxicity (16). However, $\sim 90 \%$ of dosed 5-FU is metabolized to $\alpha$-fluoro- $\beta$ alanine by dihydropyrimidine dehydrogenase (DPD) in liver, which causes hand-foot syndrome and neurotoxicity $(16,18,19)$. This metabolism by DPD prevents the drug's antitumor effect. In this study, i.p. injections were performed as a systemic administration, since we previously demonstrated that i.p. administration is available for the systemic treatment instead of i.v. injection in animal xenograft models (20), and here, no organ toxicity was found with M-DDS infusion of 5-FU, although toxicity in several organs was found with daily i.p. injections of 5-FU. These results indicate that antitumor activity of the drug might not be inhibited by liver metabolism when administered via M-DDS and that various toxicities are ameliorated, producing a desirable antitumor effect.

Direct and local delivery of an anticancer drug to a tumor is expected to offer strong antitumor activity since a high concentration of the drug reaches the tumor region. Although this strategy has been studied for the treatment of pancreatic tumors since the 1960s (21-24), these studies have failed to achieve their primary focus of prolonging the lifetime of the patients (25-27). There are several advantages of a novel M-DDS approach as proposed in this study: i) the infusion rate or drug contents can be altered during an experiment at any time; ii) the high infusion pressure can allow continuous infusion of solution at a constant rate, independent of circumstances at the catheter end; iii) the pump is silent, and therefore causes less stress to the host; iv) the pump has a battery of life of 2 weeks or more; and v) the receiver consists of fluorine material which tolerates chemicals, and, in addition, the residual quantity of drug can be checked at any time. As results of this study, we aim to define the standard for discrimination between some drugs, which are adequate for the sustained treatment as 5-FU and other drugs, which are not proper for it, and then to develop the risk benefit balances of antitumor agents by M-DDS to improve the quality of life for the cancer patients.

In conclusion, a novel M-DDS consisting of a control circuit and drug reservoir/pump unit may be a useful tool for the treatment of not only pancreatic cancer but also various accessible cancers without effective treatment, such as bileduct and brain tumors. To utilize this M-DDS in clinical trials, currently we are evaluating the safety and feasibility of the M-DDS for use in the human body.

\section{Acknowledgements}

We thank Ritsuko Asai for expert assistance with the animal experiments. This study was in part supported by a 
fellowship from the Japan Human Science Foundation (to Mari Haramoto).

\section{References}

1. Jemal A, Siegel R, Ward E, et al: Cancer statistics. CA Cancer J Clin 57: 43-66, 2007.

2. Burris HA, Moore MJ, AndersonJ, et al: Improvements in survival and clinical benefit with gemcitabine as first-line therapy for patients with advanced pancreas cancer: a randomized trial. J Clin Oncol 15: 2403-2413, 1997

3. Piccart-Gebhart MJ, Procter M, Leyland-Jones B, et al: Trastuzumab after adjuvant chemotherapy in HER2-positive breast cancer. N Engl J Med 353: 1659-1672, 2005.

4. Heist RS and Christiani D: EGFR-targeted therapies in lung cancer: predictors of response and toxicity. Pharmacogenomics 10: 59-68, 2009

5. Kawakami K, Terabe M, Kioi M, et al: Intratumoral therapy with IL 13-PE38 results in effective CTL-mediated suppression of IL-13R alpha2-expressing contralateral tumors. Clin Cancer Res 12: 4678-4686, 2006.

6. Kawakami K, Kawakami M, Husain SR and Puri RK: Targeting interleukin-4 receptors for effective pancreatic cancer therapy. Cancer Res 62: 3575-3580, 2002.

7. Kawakami K, Nakajima O, Morishita R and Nagai R: Targeted anticancer immunotoxins and cytotoxic agents with direct killing moieties. Scientific World J 6: 781-790, 2006.

8. Hamaguchi T, Matsumura Y, Suzuki M, et al: NK105, a paclitaxel-incorporating micellar nanoparticle formation, can extend in vivo antitumour activity and reduce the neurotoxicity of paclitaxel. Br J Cancer 92: 1240-1246, 2005.

9. LaVan AD, McGuire T and Langer R: Small-scale systems for in vivo drug delivery. Nat Biotechnol 21: 1184-1191, 2003.

10. Cooper JD, Heppert KE, Davies MI and Lunte SM: Evaluation of an osmotic pump for microdialysis sampling in an awake and untethered rat. J Neurosci Methods 160: 269-275, 2007.

11. Fujioka K, Maeda M, Hoji T and Sano A: Protein release from collagen matrices. Adv Drug Deliv Rev 31: 247-266, 1998.

12. Kawakami K, Husain SR, Kawakami M and Puri RK: Improved anti-tumor activity and safety of interleukin-13 receptor targeted cytotoxin by systemic continuous administration in head and neck cancer xenograft model. Mol Med 8: 487-494, 2002.

13. Feng N, Jin H, Wang M, et al: Antitumor activity of virulizin, a novel biological response modifier (BRM) in a panel of human pancreatic cancer and melanoma xenografts. Cancer Chemother Pharmacol 51: 247-255, 2003.
14. Nakamura J, Horimoto T, Hirayama R, et al: Effect of the absorption enhancer saponin on the intrarenal distribution of 5-Fluorouracil following its kidney surface application in rats. Biol Pharm Bull 26: 1049-1051, 2003.

15. Heidelberger C, Chaudhuri NK, Danneberg P, et al: Fluorinated pyrimidines, a new class of tumour-inhibitory compounds. Nature 179: 663-666, 1957.

16. Pinedo HM and Peters GF: Fluorouracil: biochemistry and pharmacology. J Clin Oncol 6: 1653-1664, 1988.

17. Skipper HE, Schabel FM Jr and Wilcox WS: Experimental evaluation of potential anticancer agents. XIII. On the criteria and kinetics associated with 'curability' of experimental leukemia. Cancer Chemother Rep 35: 1-111, 1964.

18. Lokich JJ, Ahlgren JD, Gullo JJ, et al: A prospective randomized comparison of continuous infusion fluorouracil with a conventional bolus schedule in metastatic colorectal carcinoma: a Mid-Atlantic Oncology Program Study. J Clin Oncol 7: 425-432, 1989.

19. Heggie GD, Sommadossi J, Cross DS, et al: Clinical pharamacokinetics of 5-fluorouracil and its metabolites in plasma, urine, and bile. Cancer Res 47: 2203-2206, 1987.

20. Kawakami K, Kawakami M, Joshi BH and Puri RK: Interleukin-13 receptor-targeted cancer therapy in an immunodeficient animal model of human head and neck cancer. Cancer Res 61: 6194-6200, 2001.

21. Bernhardt LC, Wirtanen GW and Ansfield FJ: Hepatic artery and eliac axis infusion for the treatment of upper abdominal malignancies. Rev Surg 26: 68, 1969.

22. Gazet JC and Smith R: Intra-arterial chemotherapy for patients with inoperable carcinoma of the pancreas. Proc R Soc Med 67: 1182-1183, 1974.

23. Smith L and Gazet J: Intra-arterial chemotherapy for patients with inoperable carcinoma of the pancreas. Ann R Coll Surg Engl 62: 208-212, 1980.

24. Theodors A, Bukowski R, Hewlett J, et al: Intermittent regional infusion of chemotherapy for pancreatic adenocarcinoma. Phase I and II pilot study. Am J Clin Oncol 5: 555-558, 1982.

25. Maurer CA, Borner MM, Läuffer J, et al: Celiac axis infusion chemotherapy in advanced nonresectable pancreatic cancer. Int J Pancreatol 23: 181-186, 1998.

26. Shibuya K, Nagata Y, Itoh T, et al: Transcatheter arterial infusion therapy in the treatment of advanced pancreatic cancer: a feasibility study. Cardiovasc Intervent Radiol 22: 196-200, 1999.

27. Aigner KR: Intra-arterial infusion: overview and novel approaches. Semin Surg Oncol 14: 248-253, 1998. 